\title{
Energy and End to End Delay Computation across Step Network under Distributed Environment
}

\author{
Kamlesh Kumar Verma \\ Department of Computer Science \\ Babasaheb Bhimrao Ambedkar University \\ Raebareli Road, Vidya Vihar \\ Lucknow (UP) 226025, India
}

\author{
Vipin Saxena \\ Department of Computer Science \\ Babasaheb Bhimrao Ambedkar University \\ Raebareli Road, Vidya Vihar \\ Lucknow (UP) 226025, India
}

\begin{abstract}
Distributed computing is widely used by many researchers due to several advantages in terms of low cost with high efficiency. In the present paper, a step topological structure is explained and process model is designed through Markov chain. The energy is computed when information is transmitted from one node to another node. End to End delay is also computed in terms of the transmitted rate. Transmission time is also computed and results are represented in terms of table and graph. Since, it is newly developed topological structure under distributed environment, hence comparison on the said topological structure is not possible however it can be converted in the form of bus topology.
\end{abstract}

\section{Keywords}

Distributed Computing, Step Network, Energy Computation, Transmission Rate, Processing Time.

\section{INTRODUCTION}

Distributed computing system is well known computing system and it has several advantages over the centralized computing system in terms of cost and efficiency. A lots of research work is available on the distributed computing systems however less number of research papers are available for the step network which is a recently designed topological structure. Let us describe some of the important research papers related to the present work. Chunlin, et al. [1] have described an algorithm to find the quality of service (QoS) and also described energy aware scheduling algorithm (QESA) for Joint optimization of network (i.e. QoS and energy conservation in grid and distribution network). Behere et al. [2] have given some technology based theory depended on cooling devices like as variable frequency drives (VFD's), blanking panels, high efficiency plug fans, and other electro mechanically devices. Authors have given control strategy wireless and wired sensors, optimization of temperature set points, through put sequence, VFD speeds, and air reheat and humidification controls. Limmer et al. [3] have proposed the use of OptiNum-Grid element method through simulations, Analog circuit simulations and e-beam simulations for energy optimization. Ding et al. [4] have described the method of the developments of distributed state estimation and distributed $\mathrm{H}$ filtering which are systematically reviewed. As the latest results on the distributed over sensor networks are discussed in details and some challenges are highlighted. Li et al. [5] have describedthe coordination of the energy resources for decentralized system. Energy resources are coordinated with each other system for efficient autonomous system and demand in large power the distributed networks, information exchange is indirect communications between the agents. The coordination mechanism is asynchronously scalable and robust. Chaudahri et al. [6] have found the performance of latency, bandwidth, through the experiment on Distributed Shared Memory (DSM) in multiprocessor system. The performance of the system described as distributed the performance parameter are latency of the process (1), occupancy (o), and node to network bandwidth (g). Ball et al. [7] have given a strategy for the optimization of design of Bayesian Network. Authors used a distributed network and the decomposition is formulated as a non linear network optimization. Bayesian network which is used by authors decomposed sensor networks in to a sectioned Bayesian networks. Islam et al. [8] have described an algorithm for a set S1 S2 S3 .........SN. of non trivial connecting sets based on maximize $\alpha=\mathrm{m} / \mathrm{k}$, connected devices are based on frequencies and maximize $\alpha$ to reduce the frequencies. Dhanalakshmi et al. [9] have described the energy consumption of the node by the using the Energy Conserving Advanced Optimized Link State Routing (ECAO) methodology. This methodology used MANET routing scheme. The routing scheme is based on data communication of the nodes. Chen et al. [10] have described a new type of sensor called cable sensor, this type of sensor has a rectangular shape sensing area with a processor. Authors have given the energy efficient communication algorithms for wireless cable sensor network. There are two cases for proposed algorithm, one is the reducing power via transmission power of processors during connectivity of network and another is scheduling cable sensor during network connectivity. There are two approaches which are reported to develop the distributed algorithm called DTRNG based on neighbour node connectivity graph. The second approach is the cable mode based transition algorithm which has evaluated the minimum number of active sensor to maintain K-coverage and connectivity.

\section{MARKOV CHAIN DECISION PROCESS MODEL}

Markov decision process model is used for discrete sequential data interpretation. This is a stochastic model for finding the process sequential definition. In this model, the probability distribution works on the next state which depends on the current state. A simple Markov process is, in which states can be observed directly. It may be autonomous or controlled process model by process sequencing. When the controlled processes are in actions for a distributed system then results affect on the outcomes process output. The Markov chain process model is shown below in figure 1 . 


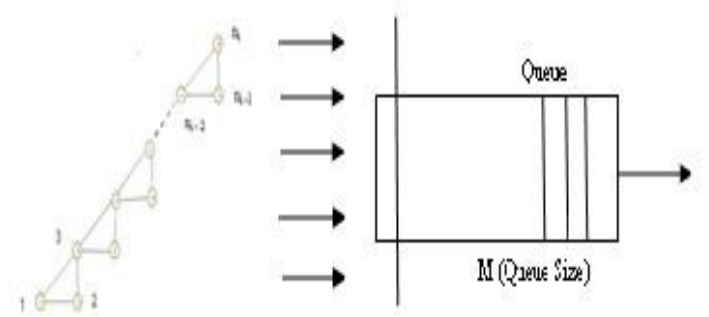

Step Netwokk Topobgy

\section{Figure 1. Markov Chain Process Model used in Step Network Topology}

Markov Decision Process (MDP) [11] as shown in figure. 2 provide a mathematical platform for modelling of the research problems which are based upon the decision making. It is a powerful analytical tools used for sequential decision under uncertainty. It is widely used in industrial and manufacturing applications. It is based upon the numbers of states in which outcomes are partly random and partly under the control of a decision results based on the decision variables. Markov Decision Process is also useful for major optimization problems solved via dynamic programming. Markov Decision Process was discovered on the 1950 . Dynamic programming is used by Markov Process. Markov process are used in different disciplines like, robotics, automated control, economics, and manufacturing. A Markov Decision Process is a discrete time stochastic control process. A Markov process is a stochastic process that operates on random sequences and outcome states according to certain probabilities condition.

Markov chains are integer time process $\lambda \mathrm{n}, \mathrm{n} \geq 0$ for which each random variable $\square$ nis integer valued and depends on the past only through most recent random variable $\lambda \mathrm{n}-1$ for all integer $n \geq 1$. $\lambda n, n \in N$ is a discrete Markov chain on state space $S=1 \ldots \ldots M$, At each time instant $t$, The system changes state, and transition. The Markov chains follow stationary property. A first order Markov chain, the Markov property states that the process state of the system at time increases $t+1$ depends only on the state of the system time t. The step topology is queued based on Markov decision process and used the node wise probability to check the process sequentially. The process outcomes are node as first priority, and then next node process waits until the first process is completed. The process outcomes are depended on the time and data frames. The process is based on FCFS (first come first serve), and time priority bases.

Probability $\quad(\lambda t+1=x t+1 \mid \lambda t=\lambda 1 \ldots \lambda t=\operatorname{Probability}(\lambda t+1=x t+1 \mid \lambda t$ $=\lambda \mathrm{t})$

A stationary equation implement with Markov property is independent of time. Then,

Probability $(\lambda t+1=\lambda i \mid \lambda t=\lambda j)=P i, j$, Where for $\forall t$ and $\forall i$, $j \in 0 \ldots N$

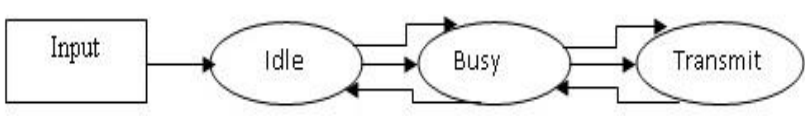

Markov decision process model

Figure 2. Markov Decision Process Model
Thus we are testing at processes whose functions are sequence of integers between 1...M. Thus Markov process is covered by transition fraction $\mathrm{Pij}$ and initial probability $\mathrm{Pi}$, Markov chains can be represented by graphs where, each state is represented by a node and directed edge represents a non zero transition probability. If a Markov chain has $M$ states then transition probability can be represented by an $\mathrm{M}$ x M matrix. The matrix $\mathrm{T}$ is stochastic matrix 1 .

The Markov model processes are based on

1. Service Type

The service types are the independent and identical.

2. Service Time

(i) It can be deterministic or exponentially distributed.

(ii) It's dependent on the queue length.

3. Service Discipline

The service disciplines are as the following:

\section{(i) FCFS}

The first come first serve process is based on time, frequency bandwidth and data packets. The service process time is minimum in FCFS and the process which comes first is automatically processed first by server.

\section{(ii) Random Order}

The random number is used by network scheduling in network topology. This random number is based on time slot. The random time slot number is defined as $\mathrm{Ti} \rightarrow \Delta \mathrm{t}$. So the time dependency depends on the random number.

\section{(iii) Priorities Scheduling}

The all real time systems usually used the pre-emptive priority scheduling systems. A process tasks must specify the time needed, then system can be complete the task.

\section{(iv) Processor Sharing}

The processor sharing is based on the dividing the total amount of time into an equal number of process used in sharing. The total time is divided into equal slots of time to complete with each other and every process. The process sharing scheduling works as entry scheme for control policy systems.

\section{Service capacity}

The service capacity may be based on single server or a group of servers.

\section{Waiting Room}

In data transmission network finitely many cells can buffered in a switch. The determination of good buffer sizes is important factor in the design communication networks.

\section{WIRE LINE CABLE TRANSITION IN DISTRIBUTED NETWORK SYSTEMS}

The various formulae used in the work are given below:

\subsection{The Packet Delivery Ratio (PDR}

The Packet Delivery Ratio is the packet frames per millisecond. It depends on the process stateinitial state to another state) and this is a relation between received packet and packet transmission in all system component such as CPU on system hardware component and given below.

Packet Delivery Ratio $=\frac{\text { No.of Re ceived Packtes }}{\text { No.of Packets Transmitted }}$ 


\subsection{The transmission control messages}

The transmission control messages are the method of controlling unit which is based on connection establishment and maintain a network conversation during. Which application programs can exchange data, the Transmission Control Protocol works with the Internet Protocol (IP), which defines how computers send packets of data to each other. The TCP and IP are the operation on the distributed network. TCP is a connection-oriented protocol, which works on a connection is established and maintained until the application programs at each end finished exchanging messages. It breaks the data packets of the networks which can deliver, sends packets to network and accepts packets from the network and manages the data flow, to provide error-free data transmission for handling retransmission of dropped packets. In the Open SystemsInterconnection (OSI) Communication model, TCP covers layer 4 the Transport Layer, and parts of Layer 5, the Session Layer.

\subsection{Average Transmission Time}

The average transmission time is defined as the process coordinated over the packets transmission of sending and receiving data per unit time in milliseconds.

\subsection{End to End Delay}

End to End delay = Processing Time + Queuing Time + transmission Time + Propagation Time.

\section{RESULTS AND DISCUSSION}

On the basis of above problem, a calculation of processing time is shown in the table 1 . This is computed for seven nodes which are connected across step network. The links between nodes are N1N2, N2N3, N1N3, N3N4, N4N5, N3N5, N5N6, N6N7 and N5N7. The end to end delay is also recorded in the table by considering the packet size which is transmitted on the said links.

Table1.1 Computation of Processing Time and End to End Delay

\begin{tabular}{|c|c|c|c|c|c|}
\hline $\begin{array}{c}\text { Sr. } \\
\text { No. }\end{array}$ & Nodes & $\begin{array}{c}\text { Proce } \\
\text { ssing } \\
\text { Time } \\
(\mathbf{m s})\end{array}$ & $\begin{array}{c}\text { E2E } \\
\text { Delay } \\
\text { (kbps) }\end{array}$ & $\begin{array}{c}\text { Data } \\
\text { Packets } \\
\text { (kbps) }\end{array}$ & $\begin{array}{c}\text { Transm } \\
\text { ission } \\
\text { time } \\
(\mathbf{m s})\end{array}$ \\
\hline 1. & $\mathrm{~N}_{1} \mathrm{~N}_{2}$ & 0.6 & 379.42 & 62 & 5.11 \\
\hline 2. & $\mathrm{~N}_{2} \mathrm{~N}_{3}$ & 0.8 & 602.9 & 72 & 5.89 \\
\hline 3. & $\mathrm{~N}_{1} \mathrm{~N}_{3}$ & 0.4 & 1040.4 & 64 & 6.10 \\
\hline 4. & $\mathrm{~N}_{3} \mathrm{~N}_{4}$ & 0.6 & 16747.2 & 1280 & 7.25 \\
\hline 5. & $\mathrm{~N}_{4} \mathrm{~N}_{5}$ & 0.4 & 990.4 & 72 & 5.10 \\
\hline 6. & $\mathrm{~N}_{3} \mathrm{~N}_{5}$ & 0.7 & 626.07 & 64 & 6.14 \\
\hline 7. & $\mathrm{~N}_{5} \mathrm{~N}_{6}$ & 0.9 & 525.7 & 64 & 6.48 \\
\hline 8. & $\mathrm{~N}_{6} \mathrm{~N}_{7}$ & 0.7 & 14318.4 & 1280 & 7.13 \\
\hline 9. & $\mathrm{~N}_{5} \mathrm{~N}_{7}$ & 0.5 & 20480.5 & 1280 & 7.50 \\
\hline
\end{tabular}

above table, authors have taken various nodes related to the processing assumption time and data packets. The end to end Delay finding results and transmission times. The time is varying for the data packets and data packets in the transmission network layer. The network layer accepts the request then the data is flowed through the transport layer. The transmission data flow is buffered in the given time duration and process synchronization occurs at process sequences and the end to end delay of data are affected with the operation process. This is shown below in following graph.

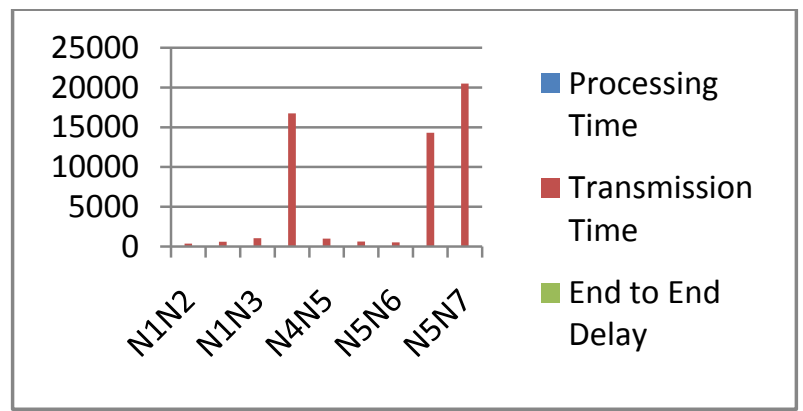

Figure 3. Relation between Processing Time, Transmission Time and End To End Delay

Let assume that,

Number of Nodes - 200

Area Size $-600 \times 600$

MAC- 802.3

Simulation Time $-50 \mathrm{sec}$

Traffic source - CBR (Constant Bit Rate)

Packet Size -512 Mbps

Transmit Power - $0395 \mathrm{w}$

Receiving Power $-0.395 \mathrm{w}$

Idle Power $-0.335 \mathrm{w}$

Initial Energy - 3.1 J

No. of Sources $-1,3,7,9$....... $\mathrm{N}_{\mathrm{i}}$

Transmission Rates $-250,500,750$, and $1000 \mathrm{kbps}$.

On the basis of above, let us generate the Cost function of the model In this model $\phi \mathrm{t}$ are the real numbers, and $\mathrm{Xt}$ are the correlated random variables $f(\mathrm{y})=\mathrm{x}_{t}$ [variable dependent for static or dynamic] $f(\mathrm{y})=\mathrm{x}_{t}\left[\phi_{s}\right.$ or $\left.\phi_{d}\right] \times$ Stationary Equation during data flow on component

$$
\begin{aligned}
& f(\mathrm{y})=\sum\left[\left(\phi_{1} x_{t-1}+\phi_{2} x_{t-2}+\ldots \ldots \ldots \ldots . . . \phi_{p} x_{t-p}+\epsilon_{t}\right) \times \lambda_{s} \text { or } \lambda_{d}\right]^{\alpha t} \\
& f(\mathrm{y})=\sum \mathrm{x}_{t} \cdot\left[\left(\lambda_{s} \text { or } \lambda_{d}\right)\right]^{\alpha t} \\
& f(\mathrm{y})=\sum \mathrm{x}_{t} \cdot\left[\left(\lambda_{s}(\mathrm{t})^{\alpha t} \text { or } \lambda_{d}(\mathrm{t})^{\alpha t}\right]+\mathrm{f}(\mathrm{w})\right.
\end{aligned}
$$

Where $\mathrm{f}(\mathrm{w})$ is a fix power consumption $f(\mathrm{y})=\int \sum x_{t} \cdot \lambda_{s}^{\alpha t}$ or $\lambda_{d}^{\alpha t}+\int f(\mathrm{w})$ 
$f(\mathrm{y})=\int_{t_{1}}^{t_{n_{i}}} \sum x_{t}+\int_{t_{n_{i}}}^{t_{T}} f(\mathrm{w})$

Total Power of the whole system,

$\mathrm{P}_{T}=\int f(\mathrm{y}) \Rightarrow \int_{t_{1}}^{t_{n_{i}}} \sum x_{t}+\int_{t_{n_{i}}}^{t_{T}} f\left(\mathrm{w}_{i}\right)$

$\operatorname{Pr}(\mathrm{x})=\operatorname{pr}\left(\mathrm{x}_{\mathrm{ni}}, \mathrm{x}_{\mathrm{n}}\right)$

$\operatorname{pr}(\mathrm{x})=\operatorname{pr}\left(\mathrm{x}_{n_{i}}, \mathrm{x}_{n_{i-1}}\right.$ $\left.\mathrm{X}_{n_{1}}\right)$

$\operatorname{pr}(\mathrm{x})=\operatorname{pr}\left(\mathrm{x}_{n_{i}}, \mathrm{x}_{n_{i-1}} \ldots \ldots \ldots \ldots \ldots . . \mathrm{x}_{n_{1}}\right) \cdot \operatorname{pr}\left(x_{n_{i-1}} / x_{n_{i-2}} \ldots \ldots \ldots \ldots . . \mathrm{x}_{n_{i}}\right)$

$\operatorname{Pr}\left(\mathrm{N}_{1} N_{2} N_{3}\right)=\operatorname{pr}\left(\mathrm{N}_{1}\right) \operatorname{pr}\left(\frac{N_{2}}{N_{1}}\right) \operatorname{pr}\left(\frac{N_{3}}{N_{1}}\right) \operatorname{pr}\left(\frac{N_{3}}{N_{3}}\right)$

(7)
$\operatorname{Pr}\left(\mathrm{N}_{3} N_{4} N_{5}\right)=\operatorname{pr}\left(\mathrm{N}_{3}\right) \operatorname{pr}\left(\frac{N_{4}}{N_{3}}\right) \operatorname{pr}\left(\frac{N_{5}}{N_{3}}\right) \operatorname{pr}\left(\frac{N_{5}}{N_{4}}\right) \operatorname{pr}\left(\frac{N_{5}}{N_{5}}\right)$

$\operatorname{Pr}\left(\mathrm{N}_{5} N_{6} N_{7}\right)=\operatorname{pr}\left(\mathrm{N}_{5}\right) \operatorname{pr}\left(\frac{N_{6}}{N_{5}}\right) \operatorname{pr}\left(\frac{N_{7}}{N_{6}}\right) \operatorname{pr}\left(\frac{N_{5}}{N_{4}}\right) \operatorname{pr}\left(\frac{N_{7}}{N_{7}}\right)$

$\operatorname{Pr}\left(\mathrm{N}_{i-2} N_{i-1} N_{i}\right)=\operatorname{pr}\left(\mathrm{N}_{i-2}\right) \operatorname{pr}\left(\frac{N_{i-1}}{N_{i-2}}\right) \operatorname{pr}\left(\frac{N_{i}}{N_{i-1}}\right) \operatorname{pr}\left(\frac{N_{i}}{N_{i}}\right)$

Node Wise Probability Distribution, Energy is given below:

\section{NODE DISTRIBUTION ENERGY}

The node wise distribution of energy is given by table 2 .

Table 2. Node Wise Distribution of Energy

\begin{tabular}{|c|l|c|c|c|c|}
\hline $\begin{array}{c}\text { Sr } \\
\text { No. }\end{array}$ & $\begin{array}{l}\text { Probability } \\
\text { Distribution(Node) }\end{array}$ & $\begin{array}{c}\text { Estimated } \\
\text { Nodes }\end{array}$ & $\begin{array}{c}\text { Node } \\
\text { Values }\end{array}$ & $\begin{array}{c}\text { Distributed } \\
\text { Energy }\end{array}$ & $\begin{array}{c}\text { Percentage Nodes } \\
\text { Wise Energy ( } \%)\end{array}$ \\
\hline 1. & $\operatorname{Pr}\left(\mathrm{x}_{i}=N_{1} / N_{i-1}\right)$ & $\mathrm{N}_{1}$ & 0.6 & $120.4 \mathrm{kw}$ & $30.89 \%$ \\
\hline 2. & $\operatorname{Pr}\left(\mathrm{x}_{i}=N_{2} / N_{i-1}\right)$ & $\mathrm{N}_{2}$ & 0.8 & $796.48 \mathrm{kw}$ & $47.84 \%$ \\
\hline 3. & $\operatorname{Pr}\left(\mathrm{x}_{i}=N_{3} / N_{i-1}\right)$ & $\mathrm{N}_{3}$ & 0.7 & $640.00 \mathrm{kw}$ & $21.26 \%$ \\
\hline 4. & $\operatorname{Pr}\left(\mathrm{x}_{i}=N_{4} / N_{i-1}\right)$ & $\mathrm{N}_{4}$ & 0.6 & $700.48 \mathrm{kw}$ & $96.42 \%$ \\
\hline 5. & $\operatorname{Pr}\left(\mathrm{x}_{i}=N_{5} / N_{i-1}\right)$ & $\mathrm{N}_{5}$ & 0.9 & $768.0 \mathrm{kw}$ & $.361 \%$ \\
\hline 6. & $\operatorname{Pr}\left(\mathrm{x}_{i}=N_{5} / N_{i-1}\right)$ & $\mathrm{N}_{6}$ & 0.4 & $120.4 \mathrm{~kW}$ & $8.22 \%$ \\
\hline 7. & $\operatorname{Pr}\left(\mathrm{x}_{i}=N_{6} / N_{i-1}\right)$ & $\mathrm{N}_{7}$ & 0.9 & $700.41 \mathrm{~kW}$ & $91.36 \%$ \\
\hline 8. & $\operatorname{Pr}\left(\mathrm{x}_{i}=N_{i} / N_{i-1}\right)$ & $\mathrm{N}_{i}$ & $\phi$ & & \\
\hline
\end{tabular}

$\operatorname{Pr}\left(N_{5} \mathrm{~N}_{6} \mathrm{~N}_{7}\right)=a_{n_{5}} \times e_{1}($ packet frame $)+a_{n_{6}} \times e_{2}($ packet frame $)+a_{n_{7}} \times \mathrm{e}_{3}($ packet frame $)$

$\operatorname{Pr}\left(N_{3} \mathrm{~N}_{4} \mathrm{~N}_{5}\right)=a_{n_{3}} \times e_{1}($ packet frame $)+a_{n_{4}} \times e_{2}($ packet frame $)+a_{n_{5}} \times \mathrm{e}_{3}($ packet frame $)$

$\operatorname{Pr}\left(N_{5} \mathrm{~N}_{6} \mathrm{~N}_{7}\right)=a_{n_{5}} \times e_{1}($ packet frame $)+a_{n_{6}} \times e_{2}($ packet frame $)+a_{n_{7}} \times \mathrm{e}_{3}$ (packet frame)

We find that, every node power utilization in networks

$\operatorname{Pr}\left(\mathrm{N}_{1} \mathrm{~N}_{2} \mathrm{~N}_{3}\right)=120.4 \mathrm{kw}$

$\operatorname{Pr}\left(\mathrm{N}_{3} \mathrm{~N}_{4} \mathrm{~N}_{5}\right)=796.48 k w$

$\operatorname{Pr}\left(\mathrm{N}_{5} \mathrm{~N}_{6} \mathrm{~N}_{7}\right)=700.48 \mathrm{kw}$

$\operatorname{Pr}\left(\mathrm{N}_{1}\right)=\frac{\operatorname{Pr}\left(\mathrm{N}_{1}\right)}{\operatorname{Pr}\left(\mathrm{N}_{1} \mathrm{~N}_{2} \mathrm{~N}_{3}\right)} \times 100$

$$
\begin{aligned}
& \operatorname{Pr}\left(\mathrm{N}_{2}\right)=\frac{\operatorname{Pr}\left(\mathrm{N}_{2}\right)}{\operatorname{Pr}\left(\mathrm{N}_{1} \mathrm{~N}_{2} \mathrm{~N}_{3}\right)} \times 100 \\
& \operatorname{Pr}\left(\mathrm{N}_{3}\right)=\frac{\operatorname{Pr}\left(\mathrm{N}_{3}\right)}{\operatorname{Pr}\left(\mathrm{N}_{1} \mathrm{~N}_{2} \mathrm{~N}_{3}\right)} \times 100 \\
& \operatorname{Pr}\left(\mathrm{N}_{4}\right)=\frac{\operatorname{Pr}\left(\mathrm{N}_{4}\right)}{\operatorname{Pr}\left(\mathrm{N}_{3} \mathrm{~N}_{4} \mathrm{~N}_{5}\right)} \times 100 \\
& \operatorname{Pr}\left(\mathrm{N}_{5}\right)=\frac{\operatorname{Pr}\left(\mathrm{N}_{5}\right)}{\operatorname{Pr}\left(\mathrm{N}_{3} \mathrm{~N}_{4} \mathrm{~N}_{5}\right)} \times 100 \\
& \operatorname{Pr}\left(\mathrm{N}_{6}\right)=\frac{\operatorname{Pr}\left(\mathrm{N}_{5}\right)}{\operatorname{Pr}\left(\mathrm{N}_{3} \mathrm{~N}_{4} \mathrm{~N}_{5}\right)} \times 100 \\
& \operatorname{Pr}\left(\mathrm{N}_{7}\right)=\frac{\operatorname{Pr}\left(\mathrm{N}_{5}\right)}{\operatorname{Pr}\left(\mathrm{N}_{3} \mathrm{~N}_{4} \mathrm{~N}_{5}\right)} \times 100
\end{aligned}
$$


Table 3. Energy Table per Node Consumption in

\begin{tabular}{|c|c|c|c|c|c|c|c|}
\hline \multicolumn{8}{|c|}{ Topology } \\
\hline & $E_{1}$ & $E_{2}$ & $E_{3}$ & $E_{4}$ & $E_{5}$ & $E_{6}$ & $E_{7}$ \\
\hline$N_{1}$ & 30 & $\mathbf{O}$ & $\mathbf{O}$ & $\mathbf{O}$ & $\mathbf{O}$ & $\mathbf{O}$ & $\mathrm{O}$ \\
\hline$N_{2}$ & $\mathbf{O}$ & 47 & $\mathbf{O}$ & $\mathbf{O}$ & $\mathbf{O}$ & $\mathbf{O}$ & $\mathbf{O}$ \\
\hline$N_{3}$ & $\mathbf{O}$ & $\mathbf{O}$ & 3.2 & $\mathbf{O}$ & $\mathbf{O}$ & $\mathbf{O}$ & $\mathbf{O}$ \\
\hline$N_{4}$ & $\mathbf{O}$ & $\mathbf{O}$ & $\mathbf{O}$ & 96 & $\mathbf{O}$ & $\mathbf{O}$ & $\mathbf{O}$ \\
\hline$N_{5}$ & $\mathbf{O}$ & $\mathbf{O}$ & $\mathbf{O}$ & $\mathbf{O}$ & .36 & $\mathbf{O}$ & O \\
\hline$N_{G}$ & $\mathbf{O}$ & $\mathbf{O}$ & $\mathbf{O}$ & $\mathbf{O}$ & $\mathbf{O}$ & 8.2 & $\mathbf{O}$ \\
\hline $\boldsymbol{N}_{7}$ & $\mathbf{O}$ & $\mathbf{O}$ & $\mathbf{O}$ & $\mathbf{O}$ & $\mathrm{O}$ & $\mathbf{O}$ & 91 \\
\hline
\end{tabular}

Total power consumption in step networkis $30+47+3.2+$ $96+0.36+8.2+91=275.76 \mathrm{~kW}$.

\section{CONCLUSION}

The end to end delay energy consumption in step networks which used Markov Chain is very crucial. The calculation of time estimation and data frames used during transmit time is important results. The node wise explanation and percentage is also calculated. In the present work, the input time and data frames, which are used are limited, however one can increase the number of nodes across the step network. The sequence of processes is varied with time and optimization of energy is computed through cost function.

\section{REFERENCES}

[1] Chunlin, Li., and Layuan, Li., 2010. "Joint optimization of application QoS and energy conservation in grid environment", International Journal of Systems Science, Vol. 41, No. 9, pp. 1027-1041,

[2] Behere, Sameer., 2015."Airflow Optimization in Data Centres", Energy Engineering, Taylor \& Francis, pp. 33-54.

[3] Limmer, Steffen., Schneider, Andre., et al. 2014.services for numerical simulations and Optimizations in Grids", International Journal of Parallel, Emergent and
Distributed Systems, Taylor \& Francis, Vol. 29, No. 6, pp. 521-543.

[4] Ding, Derui. Zidong Wang \& Bo Shen., 2014."Recent Advances on Distributed Filtering for Stochastic Systems over Sensor Networks", International journal of general Systems, Taylors and Francis Vol. 43, Nos. 3- 4, pp. 372- 386

[5] Li, Jiaming., Geoffrey Poulton and Geoffrey, James. 2010 " Coordination of Distributed Energy Resource Agents". Applied Artificial Intelligence, Taylor \& Francis, pp. 351-380.

[6] Chaudhuri, Mainak., et al. 2003 "Latency, Occupancy And Bandwidth in DSM Multiprocessors: A performance Evaluation". IEEE Transactions on Computers, Vol 52, No.7.

[7] Ball, D., Yan, T., Deshmukh, A., Gao, R. X.,2008“A Strategy For Decomposing Large - Scale EnergyConstrained Sensor Networks For System Monitoring", Production Planning \& Control ,Taylor \& Francis, Vol. 19, No. 4, pp. 435- 447.

[8] Islam, Kamrul ., and Akl, Selim G., 2011“A Local Algorithm To Compute Multiple ConnectedDominating sets In Wireless Sensor Networks", International Journal of Parallel, Emergent andDistributed Systems, Taylor \& Francis,Vol. 26, No. 5, pp.369-380.

[9] Dhanalakshmi, N., and Alli, p., 2015 "Efficient EnergyConservation in MANET using Energy ConservingAdvanced Optimized Link State Routing Model", Inter.J. of Parallel, Emergent and Distributed Systems, Taylor \& Francis, Vol. 31, No .5, pp. $469-$ 480

[10] Chen, Xiao., and Rowe, Neil C., 2013 "Energy- efficient Communication in Wireless Cable Sensor Networks", International Journal of Parallel, Emergent and Distributed Systems, Vol. 28, No. 4, pp. 289-307.

[11] Ching, Wai-kai., Huang, Ximin., Ng, Michael K.,Siu, Tak-Kuen., 2013.“" Markov Chains models, Algorithms and Applications", Inter. Series in Operations Research and Manag. Science.Vol.189, Springer Science business. 